\title{
Estágios supervisionados em serviços públicos de saúde da Universidade Estadual de Londrina e as Diretrizes Curriculares Nacionais em Odontologia: uma análise documental
}

\author{
Ananda Lousada Felipe \\ Cirurgiã-dentista \\ Graduação em Odontologia - UEL \\ $\triangle$ anandafelipe@hotmail.com \\ Márcio Grama Hoeppner \\ Docente do Departamento de Odontologia Restauradora - UEL \\ Doutorado em Dentística Restauradora - UNESP \\ Mestrado em Dentística Restauradora - UNESP \\ Especialização em Docência do Ensino Superior - UNIPAR \\ Especialista em Dentística Restauradora - UNESP \\ Graduação em Odontologia - UNESP \\ هhoeppner@uel.br \\ Pablo Guilherme Caldarelli \\ Docente do Departamento de Medicina Oral e Odontologia Infantil - UEL \\ Doutorado em Odontologia (Cariologia) - UNICAMP \\ Mestrado em Odontologia em Saúde Coletiva - UNICAMP \\ Especialização em Ativação de Processos de Mudança na \\ Formação Superior de Profissionais de Saúde - FIOCRUZ \\ Especialização em Saúde Coletiva e da Família - UNICAMP \\ Especialização em Gestão em Saúde - UNIFESP \\ Graduação em Odontologia - UEL \\ $\triangle$ pablocaldarelli@uel.br
}

Recebido em 25 de agosto de 2020

Aceito em 5 de março de 2021

\section{Resumo:}

Nas Diretrizes Curriculares Nacionais (DCN) em Odontologia estão descritas as competências gerais e específicas relacionadas ao processo de formação do cirurgião-dentista. Para o cumprimento dessas competências, as DCN mencionam ser imprescindível a aproximação do profissional ao sistema de saúde vigente no país, ratificando a importância do Sistema Único de Saúde (SUS) para a formação em saúde. O objetivo do presente estudo foi analisar as atividades formativas de estágios supervisionados em serviços públicos de saúde do curso de Odontologia da Universidade Estadual de Londrina (UEL) e seu alcance em relação ao cumprimento das competências gerais das DCN. Trata-se de um estudo exploratório e descritivo, do tipo pesquisa documental, no qual foram analisadas as DCN em Odontologia, o Projeto Pedagógico e os programas de aprendizagem dos módulos das referidas atividades de ensino do curso de Odontologia da Universidade Estadual de Londrina (UEL). Foi utilizada a Análise de Conteúdo proposta por Bardin para análise dos dados. Os resultados mostram o quão convergentes encontram-se os programas de aprendizagem e as atividades práticas desenvolvidas pelos estudantes, durante a experiência dos estágios supervisionados da UEL, tendo como referência as competências gerais descritas nas DCN para os cursos de Odontologia no Brasil. Conclui-se que os estágios supervisionados em serviços públicos de saúde desempenham importante contribuição no desenvolvimento das competências descritas nas DCN. As experiências, tanto profissionais quanto pessoais, vivenciadas durante as atividades extramuros, podem ser consideradas indispensáveis para formação de cirurgiões-dentistas mais completos, aptos a desenvolverem práticas humanizadas e resolutivas e, sobretudo, mais preparados e seguros para o mercado de trabalho.

Palavras-chave: Educação Baseada em Competências, Educação em Odontologia, Currículo, Estágio, Serviços de Saúde. 


\title{
Supervised internships in public health services at the State University of Londrina and the National Curriculum Guidelines in Dentistry: document analysis
}

\begin{abstract}
:
National Curriculum Guidelines (DCN) of Dentistry courses describe the general and specific competencies related to the dental education process. In order to fulfill these competencies, DCN claim that the health professional must close experience the national health system, due to the importance of the Unified Health System (SUS) to health education. This study aimed to analyze educational activities performed in supervised internships of public health services from the Dentistry course at the State University of Londrina (UEL) and their reach in relation to the fulfillment of the DCN's general competencies. This is an exploratory and descriptive study, type documentary research that the DCN in Dentistry, the Pedagogical Project and the learning programs of the Dentistry course at UEL were analyzed based on content analysis proposed by Bardin. The results highlight the existed convergence between the teaching program and the practical activities developed by internship students at UEL, considering the general competences described in the Brazilian DCN for Dentistry courses. It is concluded that supervised internships in public health services play an important role in the development of the competencies previously described in DCN. Moreover, both professional and personal experiences acquired during external activities can be indispensable to achieve a more comprehensive dental education. As a result, dental professionals can be able to develop humanized and resolutive practices, as well as be more prepared and safer for the professional market in dental field.
\end{abstract}

Keywords: Competency-Based Education, Education, Dental, Curriculum, Clerkship, Health Service.

\section{Pasantías supervisadas en servicios de salud pública en la Universidad Estatal de Londrina y las Directrices Curriculares Nacionales en Odontología: un análisis documental}

\begin{abstract}
Resumen:
Las Directrices Curriculares Nacionales (DCN) en Odontología describen las competencias generales y específicas relacionadas con el proceso de capacitación del cirujano-dentista. Para cumplir con estas competencias, las DCN mencionan que es esencial acercar a los profesionales al sistema de salud vigente en el país, lo que confirma la importancia del Sistema Único de Salud (SUS) para la capacitación en salud. El objetivo del presente estudio fue analizar las actividades de capacitación de pasantías supervisadas en servicios de salud pública del curso de Odontología en la Universidad Estatal de Londrina (UEL) y su alcance en relación con el cumplimiento de las competencias generales de las DCN. Este es un estudio exploratório y descriptivo, de tipo investigación documental, en el que se analizaron las DCN en Odontología, el Proyecto Pedagógico y los programas de aprendizaje de los módulos de las actividades docentes referidas al curso de Odontología en la Universidad Estatal de Londrina (UEL). Para el análisis de datos se utilizó el Análisis de Contenido propuesto por Bardin. Los resultados muestran cómo convergen los programas de aprendizaje y las actividades prácticas desarrolladas por los estudiantes, durante la experiencia de las pasantías supervisadas en la UEL, teniendo como referencia las competencias generales descritas en las DCN para cursos de Odontología en Brasil. Se concluye que las pasantías supervisadas en los servicios de salud pública presentan una contribución importante en el desarrollo de las competencias descritas en las DCN. Las experiencias, tanto profesionales como personales, experimentadas durante actividades fuera de la facultad, pueden considerarse indispensables para la formación de dentistas más completos, capaces de desarrollar prácticas humanizadas, resolutivas $\mathrm{y}$, sobre todo, más preparadas y seguros para el mercado laboral.
\end{abstract}

Palabras clave: Educación Basada en Competencias, Educación en Odontología, Curriculum, Servicios de Salud. 


\section{INTRODUÇÃO}

As Diretrizes Curriculares Nacionais (DCN) são um conjunto de orientações que preconizam a homogeneidade na elaboração de currículos dos cursos de graduação em todo âmbito nacional. As DCN para os cursos de Odontologia no Brasil foram publicadas em 2002, por meio do Parecer CES/CNE 1.300/2001, de 06 de novembro de 2001 e a Resolução CNE/CES 3, de 19 de fevereiro 2002 (BRASIL, 2002), enquanto que a sua atualização, aprovada em 05 de dezembro de 2018, por meio do Parecer CNE/CES № 803/2018, aguarda homologação (BRASIL, 2019). As DCN em Odontologia buscam orientar a formação de um cirurgião-dentista com perfil generalista, humanista, crítico e reflexivo, para atuar em todos os níveis de atenção à saúde, com base no rigor técnico e científico, no processo de saúde-doença e em seus diferentes níveis de atenção no Sistema Único de Saúde (SUS) (BRASIL, 2002; 2019).

Nas DCN em Odontologia encontram-se descritas competências gerais e competências específicas relacionas ao processo de formação do cirurgião-dentista. Após a atualização das DCN em Odontologia, as competências gerais estão estruturadas nos eixos: atenção à saúde, tomada de decisões, comunicação, liderança, gestão em saúde e educação permanente. Para o cumprimento dessas competências, as DCN mencionam que se torna imprescindível a aproximação do profissional ao sistema de saúde vigente no país, baseando-se na atenção integral da saúde, em um sistema regionalizado e hierarquizado de referência e contra referência, e no trabalho em equipes multi/interprofissionais, ratificando a importância do SUS para a formação de recursos humanos (EMMI; SILVA; BARROSO, 2017).

Nesse contexto, a vivência clínica extramuros, como os estágios supervisionados em serviços públicos de saúde, propicia ao estudante o contato com diferentes realidades socioeconômicas, hábitos, valores, condições de saúde, geral e bucal, além de promover a atuação em saúde coletiva, tendo o SUS como cenário real de práticas. Essas experiências apresentam significativo papel sobre os discentes, uma vez que melhoram a compreensão das questões éticas e sociais relacionadas à saúde bucal das populações; melhoram o acesso aos serviços odontológicos; aumentam a autonomia dos estudantes, além de aprimorarem as competências de gestão e habilidades técnicas. Dessa forma, a atuação em serviços públicos de saúde é imprescindível para a concretização dos objetivos almejados no processo de ensino-aprendizagem dos futuros cirurgiões-dentistas, considerando que, para aprender saúde, é preciso participar dos espaços onde se faz saúde (FAÉ et al., 2016). 
Diante disso, seguindo as conjunturas previstas pelas DCN, o curso de Odontologia da Universidade Estadual de Londrina (UEL) buscou o cumprimento dessas recomendações por meio de uma matriz curricular organizada em módulos, que permite a integração entre os conteúdos, racionalização do tempo e favorece a inserção de temas atuais e importantes para a formação de profissional consciente, comprometido e sabedor do seu papel como um profissional da saúde. Os módulos vinculados ao eixo de conhecimento "Odontologia em Saúde Coletiva" têm por objetivo possibilitar aos estudantes o trabalho em equipes multiprofissionais de saúde, além de capacitá-los para atuar em serviços públicos de saúde em diferentes níveis de complexidade, buscando reduzir o distanciamento entre a universidade/curso e o modo de prestação real dos serviços, pautado na excelência técnica e em evidências científicas (CEPE/UEL, 2005; SILVA; CALDARELLI, 2013).

Nesse eixo de conhecimento, estão os estágios supervisionados extramuros em serviços públicos de saúde. Os estágios estão alocados em dois módulos, um na quarta e outro na quinta série do curso. Na quarta série, a carga horária anual do módulo "Estágio Supervisionado em Serviços de Saúde dos Municípios da Região - Urbano e Rural" é de 120 horas, enquanto que na quinta série são disponibilizadas 238 horas para o módulo "Estágio Supervisionado em Equipe Multiprofissional". Ao todo, cerca de 120 alunos, divididos em duplas, têm a oportunidade de vivenciar, anualmente, práticas com diversificado nível de complexidade, em distintos serviços públicos de saúde, tais como: Unidades Básicas de Saúde (UBS) e Unidades de Saúde da Família (USF) urbanas e rurais, Pronto Socorro Odontológico (PSO/UEL), Clínica de Especialidades Infantis (Bebê-Clínica/UEL), Centro Cirúrgico Odontológico e Hospital Universitário (HU/UEL) (CEPE/UEL, 2005; SILVA e CALDARELLI, 2013).

Diante do exposto, o objetivo do presente estudo foi analisar as atividades formativas dos estágios supervisionados em serviços públicos de saúde do curso de Odontologia da UEL, seu alcance em relação ao cumprimento das competências gerais das DCN e suas contribuições para o processo de formação do cirurgião-dentista. 


\section{METODOLOGIA}

Trata-se de um estudo exploratório e descritivo, do tipo pesquisa documental. Nesse contexto, a pesquisa documental se caracteriza por uma sequência de operações (pré-análise, a organização do material e análise dos dados coletados) que visam estudar documentos no intuito de compreender distintas circunstancias. Essas operações buscam elucidar o conteúdo expresso nos documentos escolhidos para o corpus da pesquisa, de forma que contextualize os assuntos em busca de se inscrever em um status científico (GARCIA JUNIOR; MEDEIROS; AUGUSTA, 2017).

Dessa forma, no presente estudo foram analisados documentos oficiais relacionados aos estágios supervisionados em serviços públicos de saúde do curso de Odontologia da UEL. Para isso, foram coletados o atual Projeto Pedagógico de Curso (PPC), implantado a partir do ano de 2006 na instituição (CEPE/UEL, 2005), os programas de aprendizagem e as respectivas ementas dos módulos: "Estágio Supervisionado em Serviços de Saúde dos Municípios da Região Urbano e Rural" e Estágio Supervisionado em Equipe Multiprofissional". Além disso, documentos oficias do Ministério da Educação e do Ministério da Saúde, como as Diretrizes Curriculares Nacionais (DCN) para os cursos de Odontologia no Brasil (Parecer CES/CNE 1.300/2001, de 06 de novembro de 2001 e Resolução CNE/CES 3, de 19 de fevereiro 2002) e a sua recente atualização que aguarda homologação, a Resolução CNE/CES 803/2018, de 05 de dezembro de 2018 foram consultados (BRASIL, 2002; 2019).

Do material obtido procedeu-se a análise dos dados, na qual foram utilizados os pressupostos do método de Análise de Conteúdo proposto por Bardin, que inclui a leitura flutuante, criação de categorias de análise e a classificação dos conceitos nas categorias criadas (BARDIN, 2011). Foi realizada a leitura minuciosa de cada um dos documentos, destacando as competências gerais descritas nas DCN (atenção à saúde, tomada de decisão, comunicação, liderança, gestão em saúde e educação permanente), sistematizando e associando-as com as principais atividades propostas aos estudantes e objetivos de aprendizagem descritos nos programas dos módulos.

Para estruturação de uma discussão baseada em evidências científicas, foi realizado um levantamento bibliográfico de publicações indexadas nas bases eletrônica de dados BBO, ISI, LILACS, MEDLINE e SciELO. Foram adotados os seguintes critérios para seleção dos artigos: estudos (descritivos, observacionais, intervencionais ou revisões da literatura) publicados 
integralmente em periódicos que adotam a revisão por pares, indexados junto às bases de dados elencadas para a realização do trabalho, em idioma português, inglês ou espanhol; no período compreendido entre o ano de 2010 até o ano de 2019. Durante a seleção dos estudos, a avaliação dos títulos e dos resumos (abstracts) identificados na busca inicial foi realizada obedecendo rigorosamente aos critérios de inclusão e a temática definida para a pesquisa.

\section{RESULTADOS}

Na perspectiva de organizar e associar os resultados encontrados foi estruturado um quadro sistematizando os achados. O Quadro 1 apresenta o compilado das análises realizadas nos documentos coletados. A construção do quadro foi desenvolvida a partir do diálogo entre as competências gerais descritas nas DCN em Odontologia (coluna 1), as atividades propostas (coluna 2) e os objetivos de aprendizagem (coluna 3) descritos nos programas de aprendizagem dos módulos que contemplam os estágios supervisionados em serviços públicos de saúde no currículo do curso de Odontologia da UEL. Ressalta-se que alguns objetivos de aprendizagem estão vinculados ao desenvolvimento de várias competências e, por isso, estão alocados mais de uma vez na coluna 3 do quadro.

Os resultados apresentados no quadro mostram o quão convergentes encontram-se os programas de aprendizagem e as atividades práticas desenvolvidas pelos estudantes durante a experiência dos estágios supervisionados da UEL, tendo como referência as competências gerais descritas nas DCN para os cursos de Odontologia no Brasil. 
Quadro 1 - Desenvolvimento de competências gerais descritas nas DCN em Odontologia e os respectivos objetivos de aprendizagem e atividade propostas nos estágios supervisionados em serviços públicos de saúde do curso de Odontologia da UEL.

\begin{tabular}{|c|c|c|}
\hline $\begin{array}{l}\text { COMPETÊNCIAS } \\
\text { GERAIS (DCN) }\end{array}$ & ATIVIDADES REALIZADAS & OBJETIVOS DE APRENDIZAGEM \\
\hline Atenção à saúde & $\begin{array}{l}\text { - Acompanhamento da rotina hospitalar junto ao Hospital Universitário } \\
\text { (HU/UEL); } \\
\text { - Aprimoramento da prática em Cirurgia Oral, por meio de treinamento } \\
\text { no Centro Cirúrgico Odontológico; } \\
\text { - Atuação no atendimento de urgências odontológicas em nível de Pronto } \\
\text { Socorro Odontológico } 24 \text { horas; } \\
\text { - Inserção das normas/condutas de controle de infecção em sua prática } \\
\text { diária; } \\
\text { - Observação e participação nos procedimentos educativos e preventivos } \\
\text { em crianças de zero a cinco anos de idade, no Centro de Especialidades } \\
\text { Infantis / Bebê Clinica-UEL; } \\
\text { - Obtenção, interpretação e registro eficiente de informações relativas à } \\
\text { história médica, exame clínico e imagens; } \\
\text { - Realização de diagnóstico das doenças da boca, as alterações sistêmicas } \\
\text { das doenças bucais, os achados bucais das doenças sistêmicas, os efeitos } \\
\text { farmacológicos sobre a cavidade bucal e condições genéticas que afetam } \\
\text { a saúde bucal da população e realizar procedimentos adequados para } \\
\text { investigação, prevenção, tratamento e controle; } \\
\text { - Realização de procedimentos educativos, preventivos, curativos e } \\
\text { emergenciais nas clínicas odontológicas das UBS. }\end{array}$ & $\begin{array}{l}\text { - Aprimorar a prática em cirurgia oral no Centro Cirúrgico } \\
\text { Odontológico e conhecer a rotina hospitalar junto ao Hospital } \\
\text { Universitário; } \\
\text { - Atuar em equipes multiprofissionais e interdisciplinares de } \\
\text { saúde na rede pública de saúde (urbano e rural). } \\
\text { - Atuar no atendimento de urgências odontológicas em nível de } \\
\text { Pronto Socorro Odontológico; } \\
\text { - Observar, participar e realizar procedimentos educativos e } \\
\text { preventivos em crianças de zero a cinco anos de idade na Clínica } \\
\text { de Especialidades Infantis (Bebê-Clínica/UEL); } \\
\text { - Realizar procedimentos de diagnóstico e tratamento de lesões } \\
\text { bucais; } \\
\text { - Realizar procedimentos de educação comunitária e atuar em } \\
\text { serviços públicos de Odontologia; } \\
\text { - Realizar procedimentos educativos, preventivos, curativos e } \\
\text { emergenciais nas clínicas odontológicas. }\end{array}$ \\
\hline Tomada de Decisões & $\begin{array}{l}\text { - Análise e interpretação de resultados de pesquisas experimentais, } \\
\text { epidemiológicas e clínicas, e desta forma atuação embasada na melhor } \\
\text { evidência científica; } \\
\text { - Discernimento e controle de questões éticas e dos problemas que } \\
\text { surgirem na prática odontológica; análise e articulação dos princípios } \\
\text { éticos para incluí-los em sua prática profissional; } \\
\text { - Proposta e execução de planos de tratamento compatíveis com as } \\
\text { terapêuticas contemporâneas, com a situação médica, psicossocial e } \\
\text { econômica, e com os objetivos/valores do paciente, apresentando-os de } \\
\text { forma lógica, lúcida e compassiva; }\end{array}$ & $\begin{array}{l}\text { - Aprimorar a prática em cirurgia oral no Centro Cirúrgico } \\
\text { Odontológico e conhecer a rotina hospitalar junto ao Hospital } \\
\text { Universitário; } \\
\text { - Atuar em equipes multiprofissionais e interdisciplinares de } \\
\text { saúde na rede pública de saúde (urbano e rural). } \\
\text { - Atuar no atendimento de urgências odontológicas em nível de } \\
\text { Pronto Socorro Odontológico; }\end{array}$ \\
\hline
\end{tabular}




\begin{tabular}{|c|c|c|}
\hline & $\begin{array}{l}\text { - Solicitação e análise dos resultados de métodos/técnicas diagnósticas } \\
\text { complementares para a construção do diagnóstico; } \\
\text { - Tomada de decisão de acordo com as prioridades do paciente } \\
\text { previamente ao tratamento e pautar as etapas subsequentes. }\end{array}$ & $\begin{array}{l}\text { - Identificar etapas e condicionantes relativos ao planejamento de } \\
\text { serviços de saúde e realização de educação comunitária em } \\
\text { diferentes níveis de complexidade; } \\
\text { - Observar, participar e realizar procedimentos educativos e } \\
\text { preventivos em crianças de zero a cinco anos de idade na Clínica } \\
\text { de Especialidades Infantis (Bebê-Clínica/UEL); } \\
\text { - Realizar procedimentos de diagnóstico e tratamento de lesões } \\
\text { bucais; } \\
\text { - Realizar procedimentos de educação comunitária e atuar em } \\
\text { serviços públicos de Odontologia; } \\
\text { - Realizar procedimentos educativos, preventivos, curativos e } \\
\text { emergenciais nas clínicas odontológicas. }\end{array}$ \\
\hline Comunicação & $\begin{array}{l}\text { - Comunicação de forma clara e objetiva, com linguagem de fácil } \\
\text { entendimento para o paciente e para as equipes multiprofissionais, } \\
\text { considerando as características culturais, psicológicas e sociais do } \\
\text { paciente/comunidade; } \\
\text { - Explicação e discussão com o paciente das responsabilidades de cada um } \\
\text { para o sucesso do tratamento, assim como o tempo requerido, sequência } \\
\text { do tratamento e frequência; } \\
\text { - Identificação das expectativas do paciente em relação ao tratamento e } \\
\text { ao determinar se poderão ser atingidas, informação do mesmo sobre o } \\
\text { prognóstico do caso; } \\
\text { - Respeito aos aspectos das variadas culturas que podem afetar o } \\
\text { relacionamento profissional/paciente/pais e/ou decisões sobre o } \\
\text { tratamento; } \\
\text { - Uso adequado da linguagem científica para se comunicar com outros } \\
\text { profissionais da área de saúde em relação à saúde do } \\
\text { paciente/comunidade. }\end{array}$ & $\begin{array}{l}\text { - Atuar em equipes multiprofissionais e interdisciplinares de } \\
\text { - } \text { saúde na rede pública de saúde (urbano e rural); } \\
\text { preventivos em crianças de zero a cinco anos de idade na Clínica } \\
\text { de Especialidades Infantis (Bebê-Clínica/UEL); } \\
\text { - Realizar procedimentos de educação comunitária e atuar em } \\
\text { serviços públicos de Odontologia; } \\
\text { - Realizar procedimentos educativos, preventivos, curativos e } \\
\text { emergenciais nas clínicas odontológicas; } \\
\text { - Vivenciar a realidade do território, nos seus diferentes aspectos } \\
\text { epidemiológicos, socioeconômicos e culturais de acordo com a } \\
\text { área de abrangência da UBS, com interações com a equipe } \\
\text { multiprofissional local e também pelo contato direto com a } \\
\text { população. }\end{array}$ \\
\hline Liderança & $\begin{array}{l}\text { - Busca ativa por conhecimentos; } \\
\text { - Proatividade e iniciativa durante a atuação em equipes } \\
\text { multiprofissionais e interdisciplinares de saúde na rede pública de } \\
\text { saúde. }\end{array}$ & $\begin{array}{l}\text { - Atuar em equipes multiprofissionais e interdisciplinares de } \\
\text { saúde na rede pública de saúde (urbano e rural); }\end{array}$ \\
\hline
\end{tabular}




\begin{tabular}{|c|c|c|}
\hline & & $\begin{array}{l}\text { - Vivenciar a realidade do território, nos seus diferentes aspectos } \\
\text { epidemiológicos, socioeconômicos e culturais de acordo com a } \\
\text { área de abrangência da UBS, com interações com a equipe } \\
\text { multiprofissional local e também pelo contato direto com a } \\
\text { população. }\end{array}$ \\
\hline Gestão em Saúde & $\begin{array}{l}\text { - Aprendizagem sobre gerenciamento e administração da força de } \\
\text { trabalho, os recursos físicos, materiais e informações quando na } \\
\text { liderança de equipes de saúde e na gestão de serviços públicos ou } \\
\text { privados; } \\
\text { - Reconhecimento sobre a realidade do território (aspectos } \\
\text { epidemiológicos, socioeconômicos e culturais) da área de abrangência } \\
\text { da UBS por meio de interaçôes com a equipe multiprofissional local e } \\
\text { também pelo contato direto com a população; } \\
\text { - Vivência na rotina do SUS, suas dificuldades, deficiências, suas normas } \\
\text { e protocolos. Compreensão do gerenciamento e administração da } \\
\text { equipe multiprofissional. }\end{array}$ & $\begin{array}{l}\text { - Atuar em equipes multiprofissionais e interdisciplinares de } \\
\text { saúde na rede pública de saúde (urbano e rural); } \\
\text { - Identificar etapas e condicionantes relativos ao planejamento de } \\
\text { serviços de saúde e realização de educação comunitária em } \\
\text { diferentes níveis de complexidade; } \\
\text { - Vivenciar a realidade do território, nos seus diferentes aspectos } \\
\text { epidemiológicos, socioeconômicos e culturais de acordo com a } \\
\text { área de abrangência da UBS, com interações com a equipe } \\
\text { multiprofissional local e também pelo contato direto com a } \\
\text { população. }\end{array}$ \\
\hline
\end{tabular}

Fonte: Próprios autores 


\section{DISCUSSÃO}

Segundo as DCN, atualizadas em 05 de dezembro de 2018, por meio do Parecer CNE/CES № 803/2018, atenção à saúde requer ao profissional de saúde, dentro de seu âmbito profissional, estar apto a desenvolver ações de prevenção, promoção, proteção e reabilitação da saúde, tanto em nível individual quanto coletivo. Cada profissional deve assegurar que sua prática seja realizada de forma integrada e contínua com as demais instâncias do sistema de saúde (SUS), sendo capaz de pensar criticamente, de analisar os problemas da sociedade e de procurar soluções para os mesmos (BRASIL, 2002; 2019). Ao analisar as atividades desenvolvidas durante os estágios, observa-se que experiência possibilita uma nova visão sobre a prática odontológica, favorecendo a integralidade do serviço, a humanização da atenção, além da realização de um maior número de atividades clínicas e de procedimento coletivos em Odontologia.

Ademais, as DCN em Odontologia orientam que o trabalho dos profissionais de saúde esteja fundamentado na capacidade de tomada de decisões, visando o uso apropriado de evidências científicas, da eficácia e do custo efetividade da força de trabalho, de medicamentos, de equipamentos, de procedimentos e de práticas. Para este fim, esses profissionais devem possuir competências e habilidades para avaliar, sistematizar e decidir as condutas mais adequadas (BRASIL, 2002; 2019), conforme observado nas atividades propostas nos estágios supervisionados do curso de Odontologia da UEL (Quadro 1), dentre as quais encontram-se a análise e interpretação de resultados de pesquisas experimentais, epidemiológicas e clínicas para uma atuação embasada em evidências científicas. Diante dessa necessidade, os estágios extramuros se apresentam como meio de aprendizado para a formação de profissionais da saúde e, assim, mostram-se fundamentais para a formação de cirurgiões-dentistas com boas habilidades técnicas e clínicas, com autonomia para o enfrentamento das dificuldades da profissão e para escolher o melhor para indivíduos, famílias e comunidades (EMMI; SILVA; BARROSO, 2017).

Para GARANHANI e MARTINS DO VALLE (2012), a formação superior de profissionais da saúde deve oportunizar espaços para o autoconhecimento do estudante, bem como, das relações interpessoais no processo formativo, buscando, desse modo, a humanização do cuidado, com estímulo ao desenvolvimento pessoal e profissional. Na lógica da formação reflexiva, por meio de estágios supervisionados em diferentes cenários dos serviços públicos 
de saúde, o estudante torna-se mais confiante, também, para a tomada de decisões frente à assistência clínica odontológica em nível coletivo. Assim, o estudante que vivencia uma experiência multi/interprofissional, durante a sua formação acadêmica, passa a ter uma nova percepção sobre seus professores e preceptores, levando em consideração não apenas as questões tecnicistas da profissão, mas quão importante é o relacionamento criado entre cirurgião-dentista, usuário e demais profissionais que atuam nos serviços de saúde, na perspectiva de um atendimento mais humano, integral e de qualidade (LIMA; SOUZA, 2010).

Nessa perspectiva, sabe-se que a lógica do trabalho fragmentado em saúde é desafiante e requer mudanças na formação e na atuação dos profissionais (CALDARELLI, 2018), para que as ações uniprofissionais não prevaleçam nas práticas dos serviços de saúde (FERREIRA et al., 2009; ESCALDA; PARREIRA, 2018). PEDUZZI et al. (2016) apontam que o cuidado em saúde necessita da integração dos profissionais para que seja alcançada a integralidade da assistência. Para isso, o trabalho interprofissional, que é caracterizado por envolver membros de duas ou mais profissões com competências complementares em práticas colaborativas com objetivos em comuns (BARR e LOW, 2013), é uma modalidade de trabalho colaborativo muito potente para a transformação das práticas em saúde, que avança para além das ações multiprofissionais. 0 trabalho interprofissional em saúde busca o fortalecimento do trabalho em equipe, promovendo a integração e a colaboração entre os profissionais, tendo como enfoque as necessidades de saúde dos usuários (PEDUZZI et al., 2013).

O desenvolvimento de competências colaborativas, na perspectiva do trabalho interprofissional em saúde, exige que as experiências de Educação Interprofissional (EIP) estejam presentes ao longo da formação do cirurgião-dentista (TOMPSEN et al., 2018). Nesse movimento, a atualização das DCN aponta para esse sentido, destacando a necessidade da atuação e do trabalho em equipe interprofissional na formação em Odontologia (BRASIL, 2019). Contudo, em relação à análise das atividades formativas dos estágios supervisionados em serviços públicos de saúde do curso de Odontologia da UEL, observa-se que as práticas interprofissionais ainda são pouco evidentes e se apresentam como uma necessidade para o processo de formação do cirurgião-dentista na instituição.

Como preconizado nas DCN, os profissionais de saúde devem ser acessíveis e manter a confidencialidade das informações a eles confiadas, na interação com outros profissionais de saúde e o público em geral (BRASIL, 2002; 2019), destacando a importância do 
desenvolvimento de competências como a comunicação. Assim, ao ingressar nos estágios supervisionados em serviços públicos de saúde, o estudante passa a atuar com o cirurgiãodentista trabalhador do SUS que compõe uma equipe multiprofissional, o que possibilita adquirir a segurança em sua comunicação e aprimorar suas interações interpessoais, baseando-se em uma escuta atenta e qualificada, eticamente comprometida e interessada no reconhecimento do outro. Essa atuação possibilita o relacionamento entre as atitudes e a postura ética de todos que trabalham e compartilham aquele ambiente (EMMI; SILVA; BARROSO, 2017).

Segundo CALDAS et al. (2012), estudantes inseridos em atividades voltadas para a atenção básica à saúde, além de vivenciarem o cotidiano dos profissionais, conhecem a realidade social na qual se inserem os indivíduos e as famílias assistidas pelos serviços, permitindo a interação ativa (escuta e comunicação) com a população e os profissionais que compõem aquela equipe. Tal experiência permite o reconhecimento das reais dificuldades e necessidades enfrentadas pela equipe de saúde no processo de trabalho, fomentando a reflexão para atuação no serviço público de saúde.

No trabalho em equipe multiprofissional, os profissionais da saúde devem estar aptos a assumirem posições de liderança, sempre tendo em vista o bem-estar do indivíduo, família e comunidade. A liderança envolve compromisso, responsabilidade, empatia, habilidade para tomada de decisões, comunicação e gerenciamento de forma efetiva e eficaz (BRASIL, 2002; 2019). Dessa forma, é notável a importância dos estágios supervisionados extramuros para uma aproximação e desenvolvimento da competência de liderança (GUERRA; SPIRI, 2013). Essas experiências permitem a criação e o estabelecimento de vínculos, autonomia para resolução dos problemas e tomada de decisões, além da vivência do trabalho em equipe por meio de atividades e práticas como as descritas no Quadro 1.

Nesse contexto, ressalta-se que na experiência dos estágios em serviços públicos os estudantes se adequam à dinâmica de funcionamento de uma equipe multiprofissional de saúde. Tal fato pode ser considerado decisivo para a inserção e conduta desses futuros profissionais no mercado de trabalho, uma vez que essa vivência retrata a realidade que grande parte dos estudantes encontrará após a conclusão do curso. Essa diversificação dos cenários de aprendizagem permite também a transformação da prática dos estudantes, que 
passam a atuar sobre problemas reais, assumir responsabilidades crescentes e refletir sobre seu papel profissional e social (PINHEIRO; DE CARVALHO; DE SOUSA VIANA, 2018).

Considerando o perfil do cirurgião-dentista formado em consonância com as DCN, espera-se que esses profissionais estejam aptos a tomar iniciativas, fazer a gestão (administração e gerenciamento) da força de trabalho, dos recursos físicos e materiais e de informação, da mesma forma que devem estar aptos a serem empreendedores, gestores, empregadores ou lideranças na equipe de saúde (BRASIL, 2002; 2019). Nesse sentido, os estágios extramuros contribuem para que os estudantes possam vivenciar na prática o funcionamento e a gestão do sistema de saúde vigente no Brasil. Dessa forma, oportuniza-se aos futuros profissionais o conhecimento tanto das potencialidades quanto das fragilidades do SUS, tonando possível uma análise crítica sobre o processo de trabalho, planejamento, avaliação e gestão do sistema. Essa realidade impulsiona os graduandos à reflexão, buscando serem profissionais mais humanos e potenciais modificadores da realidade encontrada (EMMI; SILVA; BARROSO, 2017), o que pode ressignificar o seu processo de formação e a sua prática como futuro trabalhador da saúde.

A literatura aponta que existe o reconhecimento por parte do estudante de que, sem a participação do SUS em sua formação, o mesmo não teria construído uma compreensão sólida do funcionamento e dos conceitos do sistema público de saúde brasileiro (DOS SANTOS et al., 2013; LEME et al., 2015; SOLANO et al., 2017). É a partir desse reconhecimento que o estudante de Odontologia, nos estágios supervisionados, entra em contato direto com o processo de trabalho centrado no usuário do SUS e executado por equipes multiprofissionais, o que difere de uma abordagem isolada, comumente realizada em consultórios privados (BULGARELLI et al., 2014). Assim, os estágios atuam como importantes dispositivos que despertam percepções de interesse dos estudantes para uma atuação na perspectiva da saúde coletiva, em busca de fazer a diferença no serviço público de saúde.

Diante disso, LORENZETTI et al. (2014) ressaltam que faltam profissionais preparados para atuar como gestores no SUS, sobretudo devido à frágil formação técnica dos profissionais de saúde para a gestão, assim como, a descontinuada e ineficiente ação da educação permanente nesta área. Além disso, soma-se a alta rotatividade dos gestores no setor público, em função da relação com os processos partidários e eleitorais que geram a descontinuidade, permanentes recomeços e a desmotivação dos profissionais e trabalhadores. A visão global 
do gestor, a perfeita consonância com as políticas públicas de saúde e a capacidade gerencial em modelos diferentes do tradicional são grandes diferenciais para a proposição de serviço à comunidade de forma inclusiva, resolutiva e integral (EMMI; SILVA; BARROSO, 2017). Por isso, reafirma-se a importância do desenvolvimento de habilidades e competências relacionadas à gestão dos serviços de saúde nos processos formativos, sendo os estágios supervisionados no SUS os componentes curriculares que melhor contemplam essa demanda.

As DCN em saúde indicam que os profissionais sejam capazes de aprender continuamente, tanto na sua formação quanto na sua prática. Dessa forma, os profissionais de saúde devem aprender a aprender e ter a responsabilidade e o compromisso com a sua educação e o treinamento/estágios das futuras gerações de profissionais (BRASIL, 2019). Segundo BONDÍA (2002), situar os estudantes no mundo do trabalho e apoiá-los na produção da reflexão sobre o que acontece na rede pública de saúde, mediando as experiências pessoais e fazendo a defesa de um sistema público de natureza universal é, sem dúvida, uma opção de natureza tecnopolítica. Nesse caso, o aprendizado mobiliza não apenas a parcela dos conhecimentos científicos e informações já acumuladas, como também cria um novo saber que é resultado da "relação entre o conhecimento e a vida humana". Esse novo saber, o autor chama de experiência, que é distinta da técnica e do trabalho, por se tratar de um saber particular, subjetivo e pessoal.

Nessa perspectiva, o diálogo entre estudante/preceptor e estudante/equipe multiprofissional torna-se um importante meio para a valorização da educação permanente, uma vez que a troca de experiências e conhecimentos leva ao reconhecimento da necessidade de estar em constante atualização, seja em relação à Odontologia ou em relação ao serviço público de saúde como um todo. MORITA et al. (2007) afirmam que a competência da educação permanente tem seus fundamentos nos pilares da educação, considerando que se baseia na necessidade do aprender constante e contínuo, deixando claro que a graduação não é um processo terminal, mas representa o início do aprendizado profissional, que deverá seguir por toda a vida. Dentre as atividades propostas nos estágios supervisionados do curso de Odontologia da UEL para viabilizar essa competência, destacam-se a busca ativa por conhecimento e o desenvolvimento de novos saberes com base no cotidiano dos serviços públicos de saúde. 
As limitações aqui encontradas estão relacionadas ao desenho do estudo em si (exploratório e descritivo, do tipo pesquisa documental), o qual não possibilita a análise da percepção e argumentação dos docentes, estudantes e preceptores envolvidos no processo dos estágios supervisionados em serviços públicos de saúde, fato considerado como limitante quando essa abordagem é adotada. Nesse sentido, os dados apresentados e discutidos nessa pesquisa devem ser melhor investigados, considerando formas alternativas de coleta e análise de dados, aprimorando a compreensão desse panorama, especialmente com a eminência da publicação da atualização das DCN para os cursos de Odontologia no Brasil.

\section{CONCLUSÃO}

As experiências, tanto profissionais quanto pessoais, vivenciadas durante as atividades extramuros, podem ser consideradas indispensáveis para formação de cirurgiõesdentistas mais completos, aptos a desenvolverem práticas humanizadas e resolutivas e, sobretudo, mais preparados e seguros para o mercado de trabalho.

Diante do exposto, conclui-se que os estágios supervisionados em serviços públicos de saúde do curso de Odontologia da UEL desempenham um importante papel no desenvolvimento e cumprimento de competências descritas nas DCN para formação do cirurgião-dentista. Entretanto, ressalta-se que a prática interprofissional em saúde se revela como uma necessidade para o processo de formação na instituição, sendo que os estágios em serviços púbicos de saúde se apresentam como espaços potenciais para o seu desenvolvimento.

\section{REFERÊNCIAS}

BARDIN, L. Análise de Conteúdo. Edições 70: São Paulo, 2011.

BARR, H.; LOW, H. Introdução à educação interprofissional [Internet]. 2013. Disponível em: https://www.observatoriorh.org/sites/default/files/webfiles/fulltext/2018/pub_caipe_intro_eip_po.pdf. Acesso em: 24 jan. 2021. 
BONDÍA, J. L. Notes on experience and the knowledge of experience. Revista brasileira de educação, n. 19, p. 20-28, 2002.

BRASIL. CNE. RESOLUÇÃO CNE/CES 3/ 2002 [internet]. Diário Oficial da União, Seção 1, P.10; Brasília, 4 de março de 2002. Disponível em: http://portal.mec.gov.br/cne/arquivos/pdf/CES032002.pdf. Acesso em: 24 jan. 2021.

BRASIL. CNE. RESOLUÇÃO CNE/CES 803/2018 [internet]. Aguardando Homologação; Brasília, 5 de dezembro de 2018. Disponível em: http://portal.mec.gov.br/docman/abril-2019-pdf/111231-pces803-18/file. Acesso em: 24 jan. 2021.

BULGARELLI, A. F.; SOUZA, K. R.; BAUMGARTEN, A.; SOUZA, J. M. d. et al. Formação em saúde com vivência no Sistema Único de Saúde (SUS): percepções de estudantes do curso de Odontologia da Universidade Federal do Rio Grande do Sul (UFRGS), Brasil. Interface-Comunicação, Saúde, Educação, v. 18, p. 351-362, 2014.

CALDARELLI, P. G. Residências multiprofissionais em saúde: um olhar ampliado para o cuidado. Revista SUSTINERE, Rio de Janeiro, v. 6, n. 1, p. 215-217, 2018.

CALDAS, J. B.; LOPES, A. C. S.; MENDONÇA, R. d. D.; FIGUEIREDO, A. et al. A percepção de alunos quanto ao programa de educação pelo trabalho para a saúde-PET-Saúde. Revista Brasileira de Educação Médica, v. 36, n. 2, p. 33-41, 2012.

CEPE/UEL. CONSELHO DE ENSINO, PESQUISA E EXTENSÃO DA UEL. Reformula o Projeto Político-Pedagógico do Curso de Odontologia, a ser implantado a partir do ano letivo de 2005. Resolução CEPE/UEL n ${ }^{\circ}$ 5/2005, de 9 de março de 2005.

DOS SANTOS, K. T.; FERREIRA, L; DE JESUS BATISTA, R.; BITENCOURT, C. T. F. et al. Percepção discente sobre a influência de estágio extramuro na formação acadêmica odontológica. Revista de Odontologia da UNESP, v. 42, n. 6, p. 420-425, 2013.

EMMI, D. T.; SILVA, D. M. C.; BARROSO, R. F. F. Experiência do ensino integrado ao serviço para formação em Saúde: percepção de alunos e egressos de Odontologia. Interface-Comunicação, Saúde, Educação, v. 22, p. 223236, 2017.

ESCALDA, P.; PARREIRA, C. M. S. F. Dimensions of interprofessional work and of collaborative practices developed at a primary care unit by a family health team. Interface-Comunicação, Saúde, Educação, v. 22(Sup. 2), p. 1717-1727, 2018.

FAÉ, J. M.; JUNIOR, M. F. S.; DE CARVALHO, R. B.; ESPOSTI, C. D. D. et al. A integração ensino-serviço em Odontologia no Brasil. Revista da ABENO, v. 16, n. 3, p. 7-18, 2016.

FERREIRA, R. C.; VARGA, C. R. R.; SILVA, R.F. Trabalho em equipe multiprofissional: a perspectiva dos residentes médicos em saúde da família. Ciência \& Saúde Coletiva, v. 14, n. 1, p. 1421-1428, 2009.

GARANHANI, M. L.; MARTINS DO VALLE, E. R. O OLHAR DO ALUNO HABITANDO UM CURRÍCULO INTEGRADO DE ENFERMAGEM: UMA ANÁLISE EXISTENCIAL. Ciência, Cuidado e Saúde, v. 10, n. 5, 2012.

GARCIA JUNIOR, E. F.; MEDEIROS, S.; AUGUSTA. C. Análise documental: uma metodologia da pesquisa para a Ciência da Informação. Revista Temática, v.13, n.7, p.138-150, 2017.

GUERRA, K. J.; SPIRI, W. C. Compreendendo o significado da liderança para o aluno de graduação em enfermagem: uma abordagem fenomenológica. Revista Brasileira de Enfermagem, v. 66, n. 3, p. 399-405, 2013. 
LeME, P. A. T.; PEREIRA, A. C.; DE CASTRO MENEGHIM, M.; MiAlHE, F. L. Perspectivas de graduandos em odontologia acerca das experiências na atenção básica para sua formação em saúde. Ciência \& Saúde Coletiva, v. 20, n. 4, p. 1255-1265, 2015.

LIMA, E. N. A.; SOUZA, E. C. F. Percepção sobre ética e humanização na formação odontológica. RGO. Revista Gaúcha de Odontologia (Online), v. 58, n. 2, p. 231-238, 2010.

LORENZETTI, J.; LANZONI, G.; ASSUITI, L.; PIRES, D. et al. Gestão em saúde no Brasil: diálogo com gestores públicos e privados. Texto Contexto Enfermagem [Internet], v. 23, n. 2, p. 417-425, 2014.

MORITA, M. C.; KRIGER, L.; CARVALHO, A. C. P.; HADDAD, A. E. Implantação das diretrizes curriculares nacionais em Odontologia. Dental Press Editora: Paraná, 2007. Disponível em: http://www.abeno.org.br/aadm/images/stories/downloads/implantacao_das_diretrizes_curriculares_nacion ais_abeno_2007.pdf. Acesso em: 24 jan. 2021.

PEDUZZI, M.; NORMAN, I. J.; GERMANI, A. C. C. G.; SILVA, J. A. M.; SOUZA, G. C. Educação interprofissional: formação de profissionais de saúde para o trabalho em equipe com foco nos usuários. Revista da Escola de Enfermagem da USP, v. 47, n. 4, p. 977-983, 2013.

PEDUZZI, M.; OLIVEIRA, M. A. C.; SILVA, J. A. M.; AGRELI, H. L. F.; MIRANDA NETO, M. V. Trabalho em equipe, prática e educação interprofissional. In: MARTINS, M. A.; CARRILHO, F. J.; ALVES, V. A. F.; CASTILHO, E. A.; CERRI, G. G. (Org.). Clínica Médica: atuação da clínica médica, sinais e sintomas de natureza sistêmica, medicina preventiva, saúde da mulher, envelhecimento e geriatria. 2. ed. Barueri: Manole, 2016. p. 171-179.

PINHEIRO, L. C. R.; DE CARVALHO, R. B.; DE SOUSA VIANA, P. F. Práticas de integração ensino-serviçocomunidade e a formação em Odontologia: possíveis conexões e fluxos no ensino na saúde. Revista da ABENO, v. 18, n. 4, p. 148-159, 2018.

SILVA, B. S.; CALDARELLI, P. G. O PET-Saúde em consonância com as Diretrizes Curriculares Nacionais de Odontologia no desenvolvimento de competências profissionais: relato de experiência. Revista da ABENO, v. 13, n. 2, p. 34-41, 2013.

SOLANO, M. P.; BULGARELLI, P. T.; DOS SANTOS, C. M.; MESTRINER, S. F. et al. Utilização de serviços de saúde bucal na atenção primária: perspectivas dos usuários do sus de um município do sul do Brasil. Revista Ciência Plural, v. 3, n. 3, p. 81-92, 2017.

TOMPSEN, N. N.; MEIRELES, E.; PEDUZZI, M.; TOASSI, R. F. C. Educação interprofissional na graduação em Odontologia: experiências curriculares e disponibilidade de estudantes. Revista de Odontologia da UNESP, v. 47, n. 5, p. 309-320, 2018.

\section{(c)) EY}

Este trabalho está licenciado com uma Licença Creative Commons - Atribuição 4.0 Internacional. 